ARTICLE

\title{
Polarized three-photon-pumped laser in a single MOF microcrystal
}

Huajun $\mathrm{He}^{1, \star}$, En Ma ${ }^{2, \star}$, Yuanjing Cui ${ }^{1, \star}$, Jiancan Yu${ }^{1}, \mathrm{Yu}$ Yang ${ }^{1}$, Tao Song ${ }^{1}$, Chuan-De Wu ${ }^{3}$, Xueyuan Chen ${ }^{2}$, Banglin Chen ${ }^{1,4} \&$ Guodong Qian ${ }^{1}$

Higher order multiphoton-pumped polarized lasers have fundamental technological importance. Although they can be used to in vivo imaging, their application has yet to be realized. Here we show the first polarized three-photon-pumped (3PP) microcavity laser in a single host-guest composite metal-organic framework (MOF) crystal, via a controllable in situ selfassembly strategy. The highly oriented assembly of dye molecules within the MOF provides an opportunity to achieve 3PP lasing with a low lasing threshold and a very high-quality factor on excitation. Furthermore, the 3PP lasing generated from composite MOF is perfectly polarized. These findings may eventually open up a new route to the exploitation of multiphoton-pumped solid-state laser in single MOF microcrystal (or nanocrystal) for future optoelectronic and biomedical applications.

\footnotetext{
${ }^{1}$ State Key Laboratory of Silicon Materials, Cyrus Tang Center for Sensor Materials and Applications, School of Materials Science and Engineering, Zhejiang University, Hangzhou 310027, China. ${ }^{2}$ Key Laboratory of Optoelectronic Materials Chemistry and Physics, Fujian Institute of Research on the Structure of Matter, Chinese Academy of Sciences, Fuzhou, Fujian 350002, China. ${ }^{3}$ Department of Chemistry, Zhejiang University, Hangzhou 310027, China.

${ }^{4}$ Department of Chemistry, University of Texas at San Antonio, San Antonio, Texas 78249-0698, USA. * These authors contributed equally to this work. Correspondence and requests for materials should be addressed to X.C. (email: xchen@fjirsm.ac.cn) or to B.C. (email: banglin.chen@utsa.edu) or to G.Q. (email: gdqian@zju.edu.cn).
} 
P olarization has been used in various fields, particularly in the field of biophotonics due to its ability to reduce multiple scattering, while to enhance the contrast and to improve tissue imaging resolution ${ }^{1-3}$. Through the measurement of the polarization state of the scattered light, a wealth of structural information of scatters (for example, lesions information in the tissue) can be collected given the fact that the microscopic structure of a scattering media is closely related to changes in the polarization state of the photon during a scattering process ${ }^{1,3}$. On the other hand, high-order multiphoton excitation can offer stronger spatial confinement, deeper tissue penetration and less Rayleigh scattering, which are significantly beneficial to the biological imaging ${ }^{4-8}$. To make use of the uniqueness of both polarization and high-order multiphoton excitation, the polarized three-photon and/or higher order pumped laser in single solid-state microcrystal is potentially useful for a new kind of biological imaging, so called multiphoton pumped (MPP) polarized emission biological imaging (Supplementary Fig. 1), but has never been realized. In order to produce such a unique laser, the gain medium not only needs to have a high multiphoton absorption (MPA) cross-section and lasing efficiency ${ }^{4,5}$, but more importantly needs to be assembled into a suitable microcavity of high concentration and orientation (especially in the case that the absorption transition moment of gain medium is anisotropic) without significant luminescent quenching to enforce the high optical gain and to generate controllable and directional laser. This is really a daunting challenge. In fact, although extensive research endeavours have been pursued to target such a goal, progress has been very slow. The initial effort to diminish the significant quenching effects on the solid state was to homogeneously disperse the gain medium such as the dye molecules with high multiphoton absorption cross-section into its solution ${ }^{6,9}$. By employing such a strategy, it still remains extremely difficult to provide with a sufficiently high quenching concentration, which limits the realization of necessary optical gain for compensating the losses. The quenching concentration means that the aggregation-caused quenching (ACQ) gradually becomes dominant when the concentration of gain medium is higher than the quenching concentration. Furthermore, the molecules in the solution are randomly oriented, which would limit their capacities to maximize the optical gain. So far, this dispersed solution methodology can only lead to the amplified spontaneous emission instead of generating threephoton or higher order pumped laser ${ }^{4,5}$. Although the luminescent properties of quantum dots are intriguing, they only have generated three-photon-pumped (3PP) random lasing in which the emission direction, position and numbers of mode frequency, and the uniformity of light-emitting region of such lasers are very difficult to control ${ }^{7,10}$. Recently, the 3PP lasing from colloidal nanoplatelets in solution has been demonstrated by Li et al. ${ }^{11}$; however, no polarization property of the 3PP lasing has been realized. Furthermore, its liquid nature has limited practical applications. To take advantage of the pore confinement of porous materials, zeolites and nanoporous silica have been explored to incorporate dye molecules and semiconducting polymers into the corresponding crystals and thin films to develop solid-state lasing ${ }^{12,13}$. However, zeolite/dye composites can only generate single-photon pumped lasing, mainly due to the incompatibility between the inorganic framework and organic guest, leading to the low loading concentration $(0.005 \sim 0.0005 \mathrm{M})$, uneven distribution of dye molecules and poor crystal morphology; while nanoporous silica/semiconducting polymer matrix basically leads to the single-photon pumped polarized amplified spontaneous emission.

Previously, we have used a porous metal-organic framework (MOF) for its pore confinement of a dye molecule bearing moderately high two-photon absorption cross-section, and realized the two-photon pumped lasing from a composite crystal bio-MOF-1 $\supset$ DMASM (DMASM $=4$ - $[p$-(dimethylamino)styryl $]$ -1-methylpyridinium) at room temperature ${ }^{14}$. However, the pores (two types of channels along the $c$-axis of about 7.0 and $10.0 \AA$, respectively) within bio-MOF-1 are still too large to exactly match the dye molecules of DMASM, thus the orientation of the dye molecules inside the pore cavities is not of a high order, particularly when the high concentration of the dye molecules are applied. Such a moderate pore confinement of bio-MOF-1 apparently has limited us to further realize the higher order multiphoton pumped laser in this solid-state crystal. In order to enhance the pore confinement efficiency of a porous MOF crystal, the pore sizes within a porous MOF need to be tuned to match the size of the dye molecule better. But the dilemma is that when the pore sizes of a porous MOF can exactly match the size of the dye molecule, the dye molecules cannot diffuse into the pore channels through the simple post-synthetic exchange process. To overcome this problem, we have developed an in situ self-assembly strategy ${ }^{15,16}$ : the components for building a MOF crystal (metal ion and organic linker) and the organic dye molecule are simultaneously assembled together to form the MOF/dye single crystals. Such a methodology has enabled us to tightly incorporate the dye molecules into the porous MOF crystals, and thus the dye molecules are highly ordered and oriented. We have also managed to immobilize high concentration of the dye molecules into the MOF crystal ZJU-68 $\supset$ DMASM ((DMASM) $)_{0.33} \mathrm{H}_{1.67}\left[\mathrm{Zn}_{3} \mathrm{O}(\mathrm{CPQC})_{3}\right], \mathrm{CPQC}$, 7-(4-carboxyphenyl)quinoline-3-carboxylate) (the average pore size of the one-dimensional channel along the $c$-axis is $6.0 \AA$ ) with the dye content over $0.4 \mathrm{M}$. Furthermore, the suitable refraction index and well-faceted MOF composite crystals of certain morphology symmetries can be naturally and efficiently utilized as the laser resonant cavities without any other fabrications. The powerful in situ self-assembly strategy, highly efficient pore confinement of ZJU-68 for DMASM dye molecule, and suitable refraction index as well as perfect crystal morphology have enabled us to target the first example of polarized three-photonpumped laser in single solid-state microcrystal.

\section{Results}

Synthesis and characterization. Reaction of a new organic linker 7-(4-carboxyphenyl)quinoline-3-carboxylic acid $\left(\mathrm{H}_{2} \mathrm{CPQC}\right)$ containing quinolone group and $\mathrm{Zn}\left(\mathrm{NO}_{3}\right)_{2} \cdot 6 \mathrm{H}_{2} \mathrm{O}$ in $\mathrm{N}, \mathrm{N}$-dimethylformamide/acetonitrile $/ \mathrm{H}_{2} \mathrm{O} / \mathrm{HBF}_{3}$ at $100{ }^{\circ} \mathrm{C}$ affords colourless hexagonal prism crystals of $\mathrm{H}_{2}\left[\mathrm{Zn}_{3} \mathrm{O}\left(\mathrm{C}_{17} \mathrm{H}_{9} \mathrm{NO}_{4}\right)_{3}\right]$ $\cdot 2.5 \mathrm{H}_{2} \mathrm{O} \cdot 0.5 \mathrm{DMF} \cdot \mathrm{MeCN}$ (ZJU-68, Fig. 1a). Single crystal X-ray diffraction studies reveal that ZJU-68 crystallizes in the $P \overline{3}$ space group (see Supplementary Table 1 for detailed crystallographic data). As shown in Fig. 2a, trinuclear secondary building units (SBUs) of $\left[\mathrm{Zn}_{3} \mathrm{O}\right]^{4+}$ are linked by the ligands $\mathrm{CPQC}^{2-}$ to form an anionic framework of $\left[\mathrm{Zn}_{3} \mathrm{O}\left(\mathrm{C}_{17} \mathrm{H}_{9} \mathrm{NO}_{4}\right)_{3}\right]^{2-}$. In this structure, nine coordination sites of $\left[\mathrm{Zn}_{3} \mathrm{O}\right]^{4+}$ are completely occupied by six carboxylates and three of nitrogen atoms from the quinoline moieties, which are different from most of metal-organic frameworks with $\left[\mathrm{M}_{3} \mathrm{O}\right]^{3 n-2}\left(n=3\right.$ for $\mathrm{M}=\mathrm{Cr}^{3+}, \mathrm{Fe}^{3+}$ and so on or $n=2$ for $\left.\mathrm{M}=\mathrm{Zn}^{2+}, \mathrm{Cu}^{2+}\right)$ SBUs in which three sites are occupied by small capping ligands such as water and hydroxide ${ }^{17,18}$.

The crystal has one-dimensional (1D) sub-nano channels along the $c$-axis with a hexagonal cross-section (Fig. 2b; Supplementary Fig. 2). The edge of the hexagon is about $3.0 \AA$. For the synthesis of laser dye functionalized crystals, we tried to introduce linearshaped laser dye cations DMASM via an ion-exchange process, as described in our previous work ${ }^{14}$, but failed. This is because the DMASM molecule (about $6.3 \AA$ in the width, Supplementary 


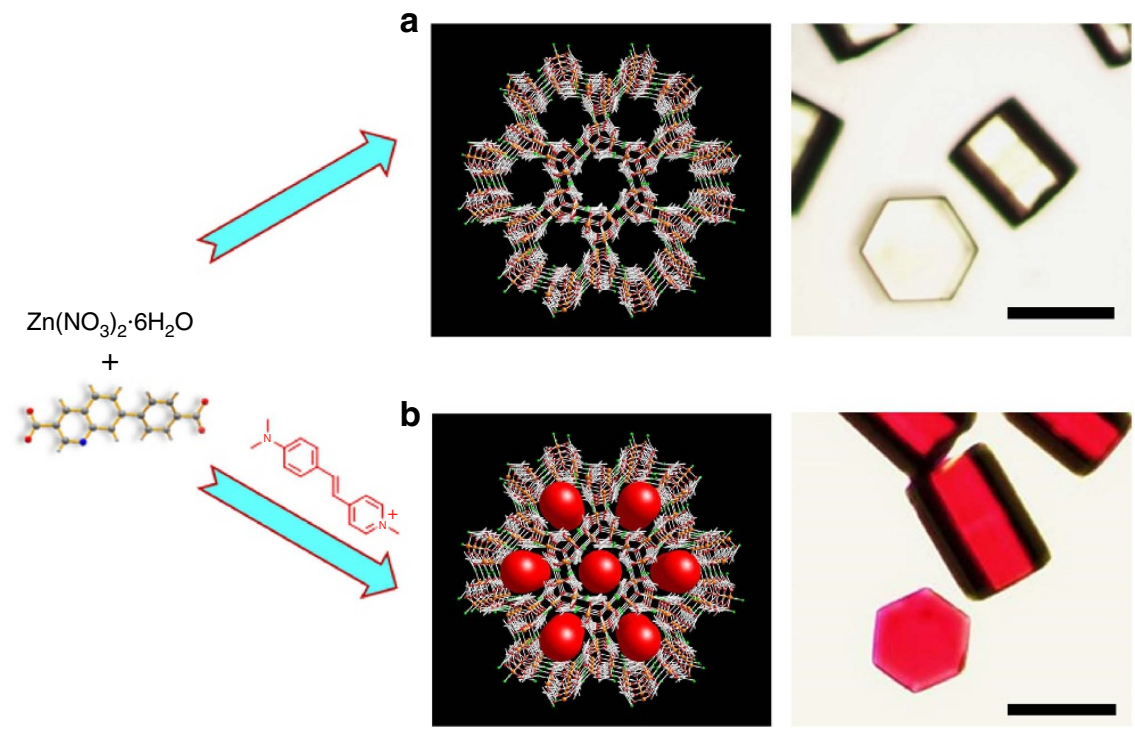

Figure 1 | Schematic synthesis of ZJU-68 and ZJU-68 $\supset$ DMASM. (a) The synthesis and micrograph of a novel metal-organic framework ZJU-68. (b) In situ synthesis of laser dye incorporated metal-organic framework crystals ZJU-68 D DMASM. The inclusion of the red dye DMASM molecules leads to the color change from the original colourless ZJU-68 to red ZJU-68 د DMASM. Scale bar, $50 \mu \mathrm{m}$.
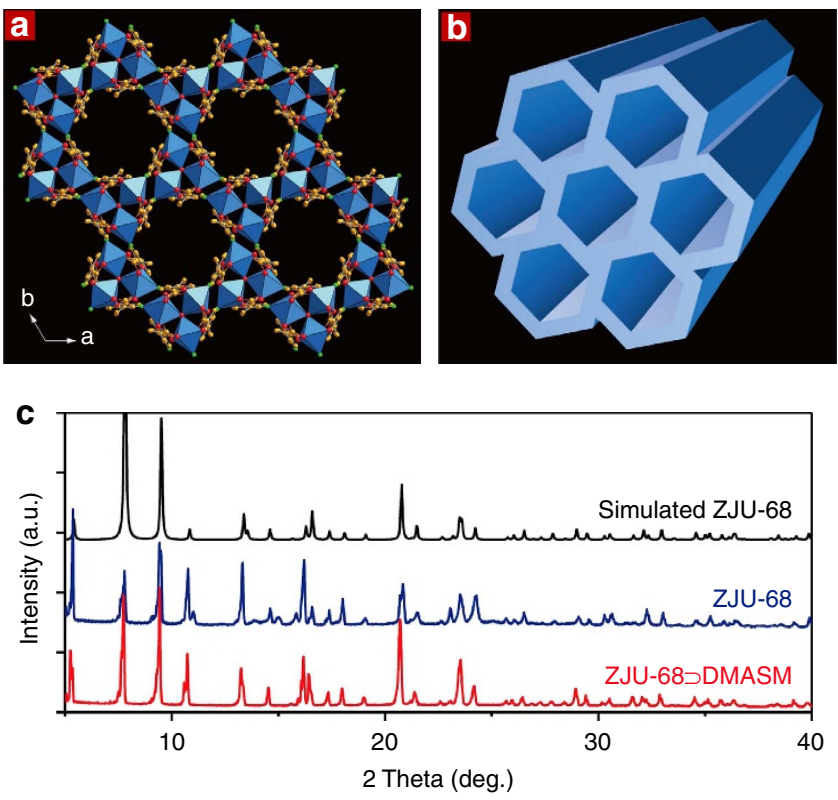

Figure 2 | The structure of a novel metal-organic framework crystal ZJU-68. (a) Crystal structure of ZJU-68 viewed along the crystallographic c direction ( $\mathrm{C}$, orange; $\mathrm{N}$, green; $\mathrm{O}$, red; $\mathrm{Zn}$, blue polyhedra). $\mathrm{H}$ atoms and solvent molecules are omitted for clarity. In this structure, nine coordination sites of a trinuclear $\mathrm{SBU}\left[\mathrm{Zn}_{3} \mathrm{O}\right]^{4+}$ are completely occupied by six carboxylates and three of nitrogen atoms from the quinoline moieties, which may play a crucial role in the stabilization of the resulting MOF, ZJU-68. (b) The simplified network structure of ZJU-68, displaying 1D channels along the $c$-axis. Different objects are not drawn to scale.

(c) PXRD patterns of ZJU-68 and ZJU-68 $\supset$ DMASM, which indicate that the ZJU-68 D DMASM has the identical framework structure with ZJU-68.

Fig. 2e) is too large to diffuse into the channels of ZJU-68 (ref. 19). We thus developed the in situ self-assembly synthetic approach in which the dye DMASM molecules were simultaneously incorporated into framework during the solvothermal synthesis of ZJU-68 by simply adding the dye molecules into the reaction solution (Fig. 1b). The resulting dye DMASM included ZJU-68 $\supset$ DMASM has the same hexagonal prism crystal morphology. The inclusion of the red dye DMASM molecules leads to the colour change from the original colourless ZJU-68 to red ZJU-68 $\supset$ DMASM. Both single crystal and the powder X-ray diffraction studies (Fig. 2c) confirmed that the ZJU-68 $\supset$ DMASM has the identical framework structure with ZJU-68. Furthermore, both ZJU-68 and ZJU-68 $\supset$ DMASM demonstrate excellent stability in the air and in the common solvents such as water, ethanol and dimethylformamide (Supplementary Fig. 3). Of course, most of the 1D hexagonal channel spaces have been occupied by DMASM molecules in ZJU-68 D DMASM. Supplementary Fig. 4 shows the fluorescence micrographs of ZJU-68 DDMASM, taken by confocal laser scanning microscope. The flat and uniform intensity profiles suggest that the DMASM dyes are homogeneously distributed inside the ZJU-68つDMASM composite crystals. The dye contents in this composite can be finely tuned by the addition of different amount of DMASM dyes during the in situ selfassembly solvothermal synthesis. Generally speaking, the relatively weak MPA responses require high dye content for MPA lasing measurements ${ }^{5,6}$, it is thus necessary to encapsulate as much dye molecules as possible into the pore space of ZJU-68. However, the high dye contents (ingredient mole ratio of $\left.n_{\mathrm{DMASM}} / n_{\mathrm{H}_{2} \mathrm{CPQC}} \geq 70 \%\right)$ in the reaction mixtures not only affect the in situ self-assembly process (formation of other MOF phases) but also lead to the formation of poor crystalline ZJU-68 $\supset$ DMASM. As such, we adjusted the dye concentration in the reaction solution, which produced the optimized ZJU-68 D DMASM crystals when the ingredient mole ratio of $n_{\text {DMASM }} / n_{\mathrm{H}_{2} \mathrm{CPQC}}$ is $35 \%$. Accordingly, per gram of resulting ZJU-68 D DMASM crystals contain $67.7 \mathrm{mg}$ dye molecules corresponding to the concentration of $0.46 \mathrm{M}$ (the molar amount of dye in per unit volume of solid composite; Supplementary Fig. 5). The optimized ingredient mole ratio $(35 \%)$ is determined by the measurement of fluorescence quantum yield. Among the ZJU-68 $\supset$ DMASM samples with different dye loading concentrations, the ZJU-68 DDMASM composite crystals with ingredient mole ratio of $35 \%$ exhibit the strongest emission at around $635 \mathrm{~nm}$ with the highest quantum 
yield $\varphi$ of $24.28 \pm 5 \%$ on excitation at $450 \mathrm{~nm}$ (Supplementary Fig. 6). This is much higher than the quantum yield of $0.45 \%$ in dye solutions and of $1.48 \%$ solid powder ${ }^{14}$. These results demonstrate that the good confinement of the DMASM molecules within the size-matched channels of ZJU-68 can effectively restrain the intramolecular torsional motion and increase the conformational rigidity of the dye, thus diminishing the ACQ and populating its radiative decay pathway ${ }^{20}$.

Multiphoton-excited fluorescence in ZJU-68 $\supset$ DMASM. Figure 3a compares the single-photon-, two-photon- and three-photon-excited fluorescence spectra of a single ZJU$68 \supset$ DMASM crystal with the dye concentration of $0.46 \mathrm{M}$ under the excitation of a femtosecond laser at different wavelengths. The ZJU-68 $\supset$ DMASM shows a strong emission peaked at $627 \mathrm{~nm}$ on excitation at $532 \mathrm{~nm}$, whereas the emission peak is red-shifted by 11 to $638 \mathrm{~nm}$ when excited at $1,064 \mathrm{~nm}$. The full-width at halfmaximum (FWHM) is 53.6 and $42.5 \mathrm{~nm}$, respectively, in the single-photon-, two-photon-excited fluorescence spectra of ZJU$68 \supset$ DMASM. The emission spectrum on excitation at $1,380 \mathrm{~nm}$ is basically similar to that excited at $1,064 \mathrm{~nm}$ except one additional small peak at around $690 \mathrm{~nm}$ attributed to the second harmonic generation response. The red shift of $11 \mathrm{~nm}$ under multiphoton excitation can be ascribed to the reabsorption effect $^{14}$. The diffuse reflectance ultraviolet-visible (vis) spectrum of ZJU-68 つ DMASM was shown in Supplementary Fig. 7. There exists overlap between the long wavelength side of the absorption band and the short wavelength side of the fluorescence band (Fig. 3a). Furthermore, all MPP fluorescence bands in Fig. 3a are asymmetric with their left part seeming to be cut off ${ }^{21}$. In addition, the emission peaked at $627 \mathrm{~nm}$ from a single ZJU$68 \supset$ DMASM crystal on excitation at $532 \mathrm{~nm}$ is blue shifted relative to the spontaneous emission (maximum at $635 \mathrm{~nm}$, see Supplementary Fig. 14) from multiple ZJU-68 つ DMASM crystals, which also suggests the presence of reabsorption effect in ZJU-68 つ DMASM (Supplementary Fig. 8). Fig. 3b shows the fluorescence intensity of the crystal with respect to the pump polarization direction when excited at $1,380 \mathrm{~nm}$. The ZJU$68 \supset$ DMASM exhibits a strong emission when the pump polarization direction is parallel to the crystal channels (along the $c$-axis, denoted as $0^{\circ}$ ), but hardly emits any light when the pump polarization direction is perpendicular to the excitation direction $\left(90^{\circ}\right)$. Such significant directional fluorescence (dicroic ratio $\sim 365$ (ref. 15)) behaviours indicate that the absorption transition moments (approximately along the dye molecule axis $^{22}$ ) are highly oriented along the crystal channels.

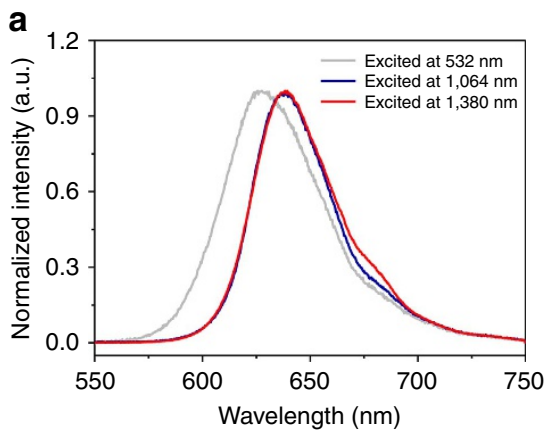

3PP lasing in ZJU-68 $\supset$ DMASM. 3PP lasing properties were investigated on an isolated single crystal of ZJU-68 $\supset$ DMASM with the dye concentration of $0.46 \mathrm{M}$ under a microscope. A femtosecond laser at $1,380 \mathrm{~nm}$ was used to pump the crystal at room temperature. This laser beam was directed from a femtosecond optical parametric amplifier (OPA), and then was coupled to the microscope. The emission beam from the crystal was focused and collected with a fibre optic spectrometer. Representative emission spectra near the lasing threshold are shown in Fig. 4a. Under the low-pump energy $(E)$ of $113 \mathrm{~nJ}$, the emission spectrum shows a broad peak centred at $\sim 649 \mathrm{~nm}$ with a FWHM of $57.6 \mathrm{~nm}$, which corresponds to the spontaneous emission. The pump energy is defined as the laser energy directly received by the MOF crystal (after going through the objective lens and before being incident on the MOF crystal). As the pump energy increases to $\geq 230 \mathrm{~nJ}$, a highly progressional emission pattern centred at $642.7 \mathrm{~nm}$ appears and grows rapidly with increasing pump energy, while the intensity of the broad spontaneous emission remains almost constant. The visible stimulated emission spectrum centred at $642.7 \mathrm{~nm}$ is between one half and one-third of the pumped wavelength of $1,380 \mathrm{~nm}$, which means that the sum energy of two photons at $1,380 \mathrm{~nm}$ is not large enough to overcome the bandgap between the ground state $\left(S_{0}\right)$ and excited state $\left(S_{1}\right)$ of ZJU-68 $\supset$ DMASM. The stimulated emission of ZJU-68 DMASM is therefore induced by the simultaneous absorption of more than two near-infrared photons. To unravel how many photons involved in such a simultaneous absorption process, we further measured the dependence of the stimulated emission intensity on the pump energy. The right inset in Fig. 4a illustrates the pump energy dependence of the fluorescence intensity and FWHM plot as a function of pump energy, giving rise to a linear relationship with cubic pump energy and a low lasing threshold of $E_{\mathrm{th}} \sim 224 \mathrm{~nJ}$ as compared with other 3PP stimulated emission $^{4,6,7}$. The FWHM plot shows a constant value below $E_{\text {th }}$ and a sudden drop by more than two orders of magnitude when above $E_{\mathrm{th}}$. The presence of a significant spectral narrowing and a threshold energy coupled with the linearly rapid increase in intensity with cubic pump energy suggest that the $3 \mathrm{PP}$ lasing has occurred in the ZJU-68 $\supset$ DMASM crystal. The quality factor $(Q)$ is given by $Q=\lambda / \delta \lambda$, where $\lambda$ and $\delta \lambda$ are the peak wavelength and its FWHM, respectively. At pump energy of $369 \mathrm{~nJ}$, the FWHM of lasing peaked at $642.7 \mathrm{~nm}$ is $\sim 0.38 \mathrm{~nm}$. This records a high-quality factor $Q \sim 1,691$ for 3PP lasing, which indicates the high crystal quality supported by our simple chemical approach without etching and coating.

For hexagonal ZJU-68 $\supset$ DMASM crystal, the opposing facets can act as the mirrors of a Fabry-Pérot (F-P) cavity, or the six

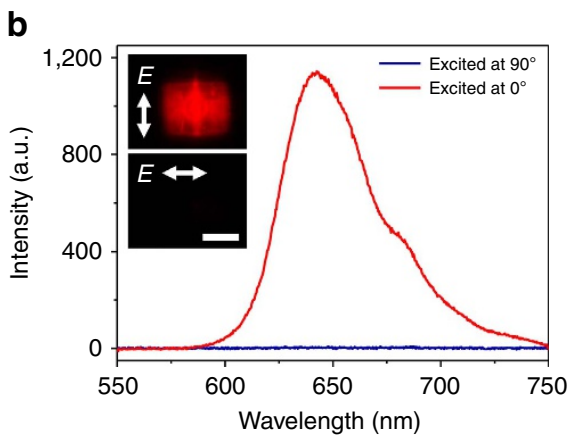

Figure 3 | Multiphoton-pumped fluorescence performance of a ZJU-68 $\supset$ DMASM single crystal. (a) Single-photon-( $532 \mathrm{~nm})$, two-photon-(1,064 nm) and three-photon- $(1,380 \mathrm{~nm})$ excited emission spectra of ZJU-68 $\supset$ DMASM. (b) The emission intensity versus pump polarization at two angles $\theta=0^{\circ}$ (parallel to the crystal channels) and $\theta=90^{\circ}$ (perpendicular to the crystal channels), excited at 1,380 nm. Insets: micrographs of a ZJU-68 $\supset$ DMASM single crystal $(R=36.5 \mu \mathrm{m})$ with different pump polarizations excited at 1,380 nm, Scale bar, $50 \mu \mathrm{m}$. The high intensity ratio between the two angles indicates the high orientation of dye molecules within the channels of ZJU-68. 

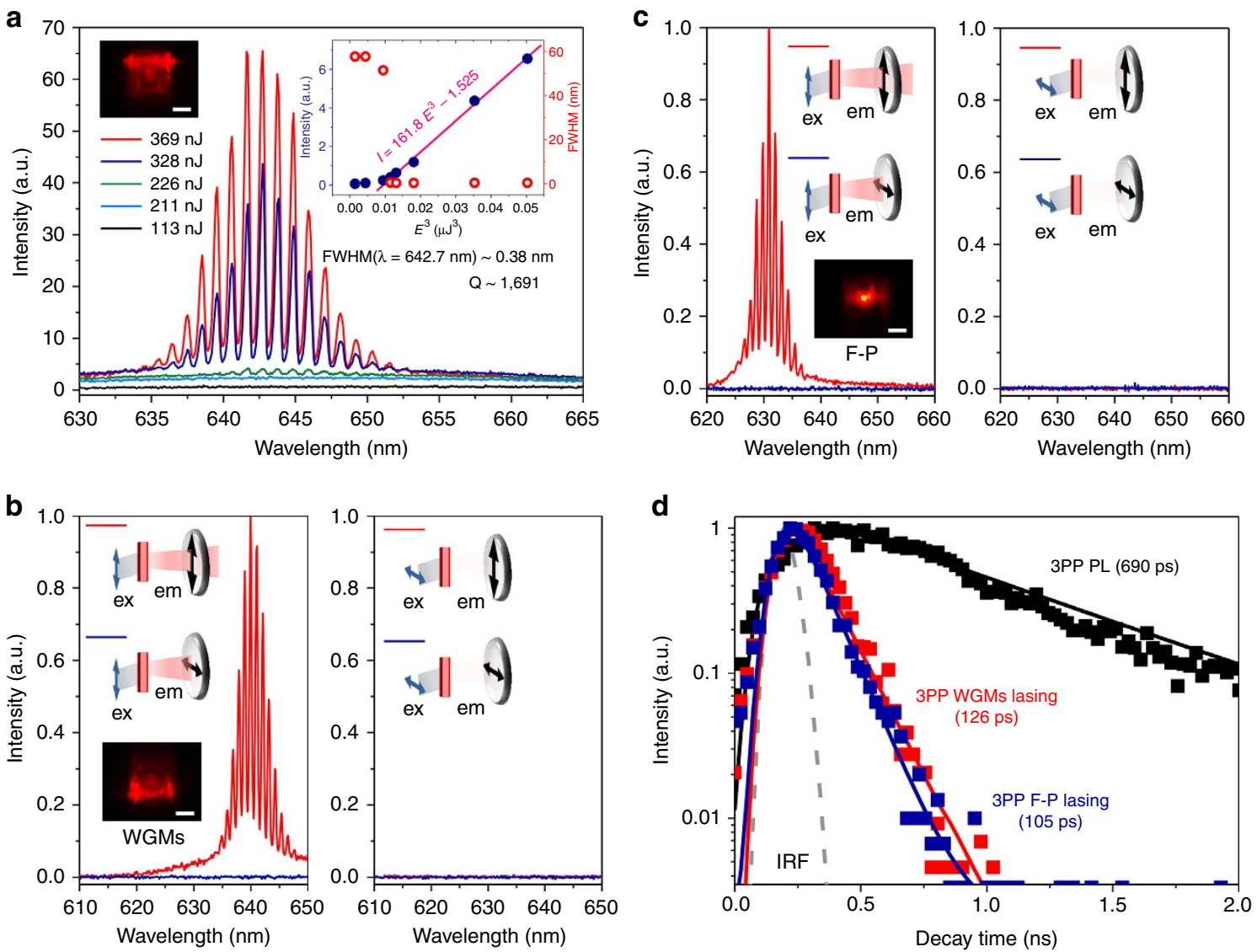

Figure 4 | Three-photon-pumped lasing performance of ZJU-68 $\supset$ DMASM. (a) 1,380 nm pumped emission spectra of ZJU-68 $\supset$ DMASM around the lasing threshold. Insets: the micrograph of a ZJU-68 D DMASM single crystal $(R=26.2 \mu \mathrm{m})$ excited at 1,380 nm (left) and emission intensity and FWHM as a function of pump energy showing the lasing threshold at $\sim 224 \mathrm{~nJ}$ (right). At pump energy of $369 \mathrm{~nJ}$, the FWHM of lasing peaked at $642.7 \mathrm{~nm}$ is $\sim$ $0.38 \mathrm{~nm}$, corresponding to a $Q$ factor $\sim 1,700$. (b,c) Intensity-dependent emission spectra of 3PP WGMs (b, pump energy at $433 \mathrm{~nJ}$ ) and F-P lasing (c, pump energy at $837 \mathrm{~nJ})$ from two isolated crystals $(R=26.1$ and $27.6 \mu \mathrm{m}$, respectively) with pump/emission-detected polarization combinations at two angles $\theta=0^{\circ}$ (parallel to the crystal channels) and $\theta=90^{\circ}$ (perpendicular to the crystal channels), excited at 1,380 nm. Insets: schematic diagrams of the measurement geometry for an individual crystal and micrographs of two kinds of lasing spot patterns due to the F-P (one-spot) and WGMs (two-spot) mechanisms, Scale bar, $20 \mu \mathrm{m}$. Both 3PP WGMs and F-P lasing exhibit perfectly polarized emission with DOP >99.9\%, which are attributed to the highly oriented assembly of dye molecules within the host-guest composite ZJU-68 $\supset$ DMASM microcrystal. (d) TRPL decay kinetics measurements of ZJU-68 D DMASM under photoluminescence (PL), F-P and WGMs lasing excited at 1,380 nm. TRPL, time-resolved photoluminescence

facets can form a whispering gallery modes (WGMs) or other quasi-WGMs cavities $^{23}$ (Supplementary Fig. 9). We observed two kinds of lasing spot pattern on isolated ZJU-68 $\supset$ DMASM crystals when excited at $1,380 \mathrm{~nm}$ : (1) the strong emission with spatial interference from two-side facets of a hexagonal prism crystal (two-spot pattern) ${ }^{24}$, as shown in the insets of microscopy image in Figs. $4 \mathrm{a}$ and $4 \mathrm{~b}$; the strong emission from a round bright spot at the central facet of the crystal (one-spot pattern) ${ }^{14}$, as shown in the inset of Fig. 4c. Such two lasing patterns can be attributed to the WGMs and F-P feedback mechanisms, respectively, as confirmed later. Fig. 4b,c show the anisotropic study of 3PP WGMs and F-P lasing from two crystals with side lengths of 26.1 and $27.9 \mu \mathrm{m}$, respectively. The red emission light from the crystal passed through a polarizer first and then was focused and collected with a fibre optic spectrometer. The schematic diagrams of the measurement geometry for an individual crystal are shown in Fig. 4b,c, where the polarization directions of the pump light and the polarizer are parallel $\left(0^{\circ}\right) /$ perpendicular $\left(90^{\circ}\right)$ to the crystal channels (along $c$-axis). We can see that both 3PP WGMs and F-P lasing with highly structured spectra occur when excited at $0^{\circ}$ and emission polarization detected at $0^{\circ}$, while hardly any emission intensity can be detected in all other configurations. The corresponding pump energy is $433 \mathrm{~nJ}$ for WGMs lasing and $837 \mathrm{~nJ}$ for F-P lasing. It should be noted that the pump energy at almost $3.2 E_{\text {th }}$ of 3PP WGMs lasing can realize the 3PP F-P lasing in our experiments, indicating that the WGMs mechanism is more conducive to the realization of $3 \mathrm{PP}$ lasing due to the total internal reflection for less loss of light in such size of the crystal. The degree of polarization can be defined as DOP $=\left(I_{\max }-I_{\min }\right) /$ $\left(I_{\max }+I_{\min }\right)$ in our experiments ${ }^{25}$, and we calculated that both 3PP WGMs and F-P lasing exhibited DOP > 99.9\% (limited by the spectral intensity sensitivity of our measurement system) when the excitation polarization is fixed parallel to the crystal channels, indicating a perfectly polarized 3PP lasing operation. Compared with the WGMs lasing, the parallel mirrors in a F-P cavity cannot be utilized as Brewster windows for the polarization selectivity. Therefore, the perfectly polarized 3PP F-P lasing is attributed to the highly oriented assembly of dye molecules within the host-guest composite ZJU-68 $\supset$ DMASM microcrystal given the fact that the angle between the absorption transition moment and emission transition moment is close to zero in the dye molecule DMASM ${ }^{26}$. These anisotropic results indicate that ZJU$68 \supset$ DMASM can only be excited at the polarization direction 
parallel to the crystal channels, and can produce lasing perfectly polarized along the crystal channels, which exhibit a great potential for bioimaging, optical sensing and future optoelectronic integration.

To further confirm the optical-feedback mechanisms for 3PP lasing in these hexagonal ZJU-68 $\supset$ DMASM crystals, several single crystals with different side lengths $R$ were chosen for the further measurements (Supplementary Fig. 10a and b). For both feedback mechanisms, the spectra of lasing exhibit an increased mode spacing with the decrease of side length of the MOF crystals. For possible resonant modes, the mode spacing $\Delta \lambda_{\mathrm{s}}$ is defined as ${ }^{27}$

$$
\Delta \lambda_{\mathrm{s}}=\frac{\lambda^{2}}{L n_{\mathrm{g}}}
$$

where $\lambda$ is the resonant wavelength, $L$ is the cavity path length ( $3 \sqrt{3} R$ for WGMs and $2 \sqrt{3} R$ for F-P cavity), and $n_{\mathrm{g}}$ is the group index of refraction. The measured mode spacing, $\Delta \lambda_{\mathrm{s}}$, around $635 \mathrm{~nm}$, demonstrates a linear relationship with $1 / R$ for each feedback mechanism, which agrees well with Equation (1) (see Supplementary Fig. 10c,d). This result indicates that the lasing with the similar output spot pattern (one-spot or two-spot) can be attributed to the same feedback mechanism. According to the fitting formula, we calculated the ratio of slopes $\left(S_{\text {one-spot }} /\right.$ $\left.S_{\text {two-spot }}\right)$ to be 1.47 , which is very close to the ratio of the cavity path lengths $\left(L_{\mathrm{WGMs}} / L_{\mathrm{F}-\mathrm{P}}=1.5\right)$, verifying that the lasing with these two kinds of output pattern (one-spot and two-spot) should be attributed to the F-P cavity and WGMs, respectively. On the basis of Equation (1), we also derived $n_{\mathrm{g}} \sim 3.27$ for F-P cavity, and $n_{\mathrm{g}} \sim 3.21$ for WGMs at the wavelength of $635 \mathrm{~nm}$. The relatively high group index $n_{\mathrm{g}}$ value may result from the unusual dispersion relation near the absorption band or the strong exciton-photon coupling in organic materials ${ }^{28}$. Further insight into the 3PP emission performance of the single crystal ZJU$68 \supset$ DMASM arises from time-resolved photoluminescence measurements (Fig. 4d). The pulse durations of the 3PP F-P lasing and WGMs lasing were determined to be 105 and $126 \mathrm{ps,}$ respectively, which are much shorter than the corresponding $3 \mathrm{PP}$ fluorescence (below the $E_{\mathrm{th}}$ ) decay time of $690 \mathrm{ps}$. Such temporal narrowing can be ascribed to the depletion in the population inversion of the gain medium with photon-stimulated amplification $^{6}$. The lasing pulse durations from WGMs and $\mathrm{F}-\mathrm{P}$ are almost in the same order of magnitude, indicating that the optical-feedback mechanism may have little effect on the pulse duration. Subtle differences in our measured decay times of lasing may be ascribed to the proportion of stimulated emission and spontaneous emission in the emitted light, which depends on multiple factors, for example, pump energy, crystal size and crystalline quality ${ }^{29,30}$.

\section{Discussion}

In summary, we have achieved an unprecedented solid-state polarized frequency-upconversion lasing in a novel composite single microcrystal ZJU-68 $\supset$ DMASM by simultaneous threephoton absorption in the near-infrared region. The tightly confined and highly oriented cationic DMASM dye molecules in anionic ZJU-68 nano-channels through an in situ assembly process efficiently increase the loaded concentration, minimize the aggregation and optimize the orientation of dye molecules within the framework, which fulfilled the high-gain lasing with highly polarized excitation response and perfectly polarized emission in a micro-sized laser cavity. Particularly, the 3PP lasing, with a low lasing threshold of $\sim 224 \mathrm{~nJ}$ centred at $642.7 \mathrm{~nm}$ on excitation at $1,380 \mathrm{~nm}$, has been successfully achieved with a record high-quality factor of $\sim 1,700$. Both F-P and WGMs optical-feedback mechanisms have been confirmed to be responsible for 3PP lasing in ZJU-68 $\supset$ DMASM microcrystals. Owing to the highly oriented assembly of dye molecules within ZJU-68 د DMASM, the 3PP WGMs and especially F-P lasing show a perfect emission polarization with DOP $>99.9 \%$. The observed solid-state frequency-upconversion polarized lasing induced by 3 PP may find great potentials in practical applications such as photonics, information storage and biomedicine, to name a few. For instance, the wavelength of $1,380 \mathrm{~nm}$ belongs to the near-infrared-IIa window $(1,300-1,400 \mathrm{~nm})$, which is very promising in biological applications (especially for in vivo imaging) because such wavelength region not only can reach deeper penetration depths and minimize the scattering/ auto-fluorescence of biological tissues, but also avoid an increased light absorption from water above $1,400 \mathrm{~nm}$ (ref. 31). Because the MOF strategy and design can provide us with rich structures of the systematically tuned pore/channel sizes to encapsulate various chromophores with controlled concentration and orientation $^{32-34}$, we anticipate that higher order multiphotonpumped lasing in solid state can also be realized given that the chromophores (or other nano-sized materials) with great multiphoton absorption properties are well incorporated into the structurally matched MOFs. These findings may eventually open up a new route to the exploitation of multiphoton-pumped solid-state laser in single MOF microcrystal (or nanocrystal) for future optoelectronic and biomedical applications.

\section{Methods}

Synthesis of ZJU-68 $\supset$ DMASM. A mixture of $\mathrm{Zn}\left(\mathrm{NO}_{3}\right)_{2} \cdot 6 \mathrm{H}_{2} \mathrm{O}(0.34 \mathrm{mmol}$, $149 \mathrm{mg}), \mathrm{H}_{2}$ CPQC (0.17 mmol, $\left.50 \mathrm{mg}\right), \mathrm{DMF}(10 \mathrm{ml}), \mathrm{MeCN}(2 \mathrm{ml}), \mathrm{H}_{2} \mathrm{O}$ $(0.05 \mathrm{ml}), \mathrm{HBF}_{3}(0.05 \mathrm{ml})$ and DMASM iodide $(0.03 \mathrm{mmol}, 11 \mathrm{mg})$ were sealed in a $15 \mathrm{ml}$ Teflon-lined stainless-steel bomb at $100^{\circ} \mathrm{C}$ for $24 \mathrm{~h}$, which was then slowly cooled to room temperature. After decanting the mother liquor, the fine red hexagonal crystalline product was rinsed three times with fresh DMF $(5 \mathrm{ml} \times 3)$ and dried in air. The synthesis of the new organic linker $\mathrm{H}_{2} \mathrm{CPQC}$ can be found in Supplementary Fig. 11 and Supplementary Methods.

Measurements. For MPP, an optical parametric amplifier (TOPAS-F-UV2, Spectra-Physics) pumped by a regeneratively amplified femtosecond Ti:sapphire laser system $(800 \mathrm{~nm}, 1 \mathrm{kHz}$, pulse energy of $4 \mathrm{~mJ}$, pulse width $<120 \mathrm{fs}$, Spitfire Pro-FIKXP, Spectra-Physics), which was seeded by a femtosecond Ti-sapphire oscillator $(80 \mathrm{MHz}$, pulse width $<70 \mathrm{fs}$, $710-920 \mathrm{~nm}$, Mai Tai XF-1, Spectra-Physics) was used for generating the excitation pulse $(1 \mathrm{kHz}, 240-2,600 \mathrm{~nm}$, pulse width $<120 \mathrm{fs}$ ). The incident laser was coupled to the microscope (Ti-U, Nikon), focusing on crystals through an objective lens (CFI TU Plan Epi ELWD $50 \times$, numerical aperture $=0.60$, work distance $=11.0 \mathrm{~mm}$ ) with an exposure region of diameter around $30 \mu \mathrm{m}$ (supplementary Fig. 12). The excited red light was then focused and collected by the fibre optic spectrometer (QE65Pro, Ocean Optics).

The decay curves of multiphoton-pumped emissions were measured by a picosecond lifetime spectrometer (Lifespec-ps, Edinburgh Instruments). For the lifetime measurement of upconverted fluorescence, the pump energy was under the lasing threshold to ensure that no stimulated emission was generated. To measure the decay of the multiphoton-pumped lasing, the pump energy was enhanced over the threshold so that the ultra-strong lasing could be achieved.

Contents of well-dried dye-included ZJU-68 $\supset$ DMASM crystals were determined by ${ }^{1} \mathrm{H}$ NMR. As shown in Supplementary Figs 5 and 13c, we calibrated and obtained peak area values of peaks that belong to $\mathrm{H}_{2} \mathrm{CPQC}$ and DMASM, respectively. The ratio $\left(R_{\mathrm{a}}\right)$ of their peak area values represents the ratio of their contents in the crystal. The dye concentration of the ZJU-68 $\supset$ DMASM composite is calculated from $c=2 R_{\mathrm{a}} / N_{\mathrm{A}} V$, where $V=2403.91 \AA^{3}$ and $N_{\mathrm{A}}=6.02 \times 10^{23} \mathrm{~mol}^{-1}$ is Avogadro's constant.

\section{References}

1. Jameson, D. M. \& Ross, J. A. Fluorescence polarization/anisotropy in diagnostics and imaging. Chem. Rev. 110, 2685-2708 (2010).

2. Ghosh, N. \& Vitkin, I. A. Tissue polarimetry: concepts, challenges, applications, and outlook. J. Biomed. Opt. 16, 110801 (2011).

3. Gurjar, R. S. et al. Imaging human epithelial properties with polarized lightscattering spectroscopy. Nat. Med. 7, 1245-1248 (2001).

4. He, G. S., Tan, L. S., Zheng, Q. \& Prasad, P. N. Multiphoton absorbing materials: molecular designs, characterizations, and applications. Chem. Rev. 108, 1245-1330 (2008). 
5. Guo, L. \& Wong, M. S. Multiphoton excited fluorescent materials for frequency upconversion emission and fluorescent probes. Adv. Mater. 26, 5400-5428 (2014).

6. Zheng, Q. D. et al. Frequency-upconverted stimulated emission by simultaneous five-photon absorption. Nat. Photon. 7, 234-239 (2013).

7. Wang, Y. et al. Stimulated emission and lasing from CdSe/CdS/ZnS core-multishell quantum dots by simultaneous three-photon absorption. Adv. Mater. 26, 2954-2961 (2014).

8. Hoover, E. E. \& Squier, J. A. Advances in multiphoton microscopy technology. Nat. Photon. 7, 93-101 (2013).

9. He, G. S., Markowicz, P. P., Lin, T. C. \& Prasad, P. N. Observation of stimulated emission by direct three-photon excitation. Nature 415, 767-770 (2002).

10. Gomes, A. S., Carvalho, M. T., Dominguez, C. T., de Araujo, C. B. \& Prasad, P. N. Direct three-photon excitation of upconversion random laser emission in a weakly scattering organic colloidal system. Opt. Express 22, 14305-14310 (2014).

11. Li, M. et al. Ultralow-threshold multiphoton-pumped lasing from colloidal nanoplatelets in solution. Nat. Commun. 6, 8513 (2015).

12. Vietze, U. et al. Zeolite-dye microlasers. Phys. Rev. Lett. 81, 4628-4631 (1998).

13. Martini, I. B. et al. Controlling optical gain in semiconducting polymers with nanoscale chain positioning and alignment. Nat. Nanotechnol. 2, 647-652 (2007).

14. Yu, J. et al. Confinement of pyridinium hemicyanine dye within an anionic metal-organic framework for two-photon-pumped lasing. Nat. Commun. 4, 2719 (2013).

15. Martinez-Martinez, V., Garcia, R., Gomez-Hortiguela, L., Perez-Pariente, J. \& Lopez-Arbeloa, I. Modulating dye aggregation by incorporation into $1 \mathrm{D}$ MgAPO nanochannels. Chemistry 19, 9859-9865 (2013).

16. Martínez-Martínez, V. et al. Highly luminescent and optically switchable hybrid material by one-pot encapsulation of dyes into MgAPO-11 unidirectional nanopores. ACS Photon. 1, 205-211 (2014).

17. Mao, C. et al. Anion stripping as a general method to create cationic porous framework with mobile anions. J. Am. Chem. Soc. 136, 7579-7582 (2014)

18. Ferey, G. et al. A chromium terephthalate-based solid with unusually large pore volumes and surface area. Science 309, 2040-2042 (2005).

19. Zhao, C. F., He, G. S., Bhawalkar, J. D., Park, C. K. \& Prasad, P. N. Newly synthesized dyes and their polymer/glass composites for one-photon and 2-photon pumped solid-state cavity lasing. Chem. Mater. 7, 1979-1983 (1995).

20. Cui, Y. J. et al. Dye encapsulated metal-organic framework for warm-white LED with high color-rendering index. Adv. Funct. Mater. 25, 4796-4802 (2015).

21. Ren, Y. et al. Synthesis, structures and two-photon pumped up-conversion lasing properties of two new organic salts. J. Mater. Chem. 10, 2025-2030 (2000).

22. Weiß, Ö. et al. in Host-Guest-Systems Based on Nanoporous Crystals 544-557 (Wiley-VCH Verlag GmbH \& Co., 2005).

23. Wang, X. et al. Whispering-gallery-mode microlaser based on self-assembled organic single-crystalline hexagonal microdisks. Angew. Chem. Int. Ed. 53, 5863-5867 (2014)

24. Braun, I. et al. Hexagonal microlasers based on organic dyes in nanoporous crystals. Appl. Phys. B 70, 335-343 (2000).

25. Zhu, H. et al. Lead halide perovskite nanowire lasers with low lasing thresholds and high quality factors. Nat. Mater. 14, 636-642 (2015).

26. Gozhyk, I. et al. Polarization properties of solid-state organic lasers. Phys. Rev. A 86, 043817 (2012).

27. Choi, S., Ton-That, C., Phillips, M. R. \& Aharonovich, I. Observation of whispering gallery modes from hexagonal $\mathrm{ZnO}$ microdisks using cathodoluminescence spectroscopy. Appl. Phys. Lett. 103, 171102 (2013).
28. Takazawa, K., Inoue, J., Mitsuishi, K. \& Takamasu, T. Fraction of a millimeter propagation of exciton polaritons in photoexcited nanofibers of organic dye. Phys. Rev. Lett. 105, 067401 (2010).

29. Zhang, C. et al. Two-photon pumped lasing in single-crystal organic nanowire exciton polariton resonators. J. Am. Chem. Soc. 133, 7276-7279 (2011).

30. Liu, X. et al. Whispering gallery mode lasing from hexagonal shaped layered lead iodide crystals. ACS Nano 9, 687-695 (2015).

31. Hong, G. S. et al. Through-skull fluorescence imaging of the brain in a new near-infrared window. Nat. Photon. 8, 723-730 (2014).

32. Furukawa, H., Cordova, K. E., O'Keeffe, M. \& Yaghi, O. M. The chemistry and applications of metal-organic frameworks. Science 341, 1230444 (2013).

33. Kitagawa, S., Kitaura, R. \& Noro, S. Functional porous coordination polymers Angew. Chem. Int. Ed. 43, 2334-2375 (2004)

34. Chen, B., Xiang, S. \& Qian, G. Metal-organic frameworks with functional pores for recognition of small molecules. Acc. Chem. Res. 43, 1115-1124 (2010).

\section{Acknowledgements}

We acknowledge the financial support from the National Natural Science Foundation of China (Nos. 51229201, 51272229, 51272231, 51402259, 51472217, 51432001, U1305244 and 21325104) and Zhejiang Provincial Natural Science Foundation of China (Nos. LR13E020001 and LZ15E020001). This work is also partially supported by Welch Foundation (AX-1730) and National Science Foundation of United States (ECCS1407443). X.C. and E.M. acknowledge the support from Special Project of National Major Scientific Equipment Development of China (No. 2012YQ120060) and the CAS/ SAFEA International Partnership Program for Creative Research Teams. We also thank Dr Ghezai Musie for proof-reading the manuscript.

\section{Author contributions}

H.H., E.M., Y.C., J.Y. and G.Q. conceived and designed the experiments. H.H. synthesized the materials. J.Y. and C.W. Analysed the crystal structure. H.H. and E.M. performed the multiphoton experiments. Y.C., J.Y., Y.Y. and T.S. assisted with the linear optical property measurements and characterization of the material. H.H., E.M., Y.C., X.C., B.C. and G.Q. analysed the data and co-wrote the manuscript. All authors discussed the results and commented on the manuscript.

\section{Additional information}

Accession codes: The X-ray crystallographic coordinates for structure reported in this study has been deposited at the Cambridge Crystallographic Data Centre (CCDC), under deposition number 1046524. These data can be obtained free of charge from the Cambridge Crystallographic Data Centre via www.ccdc.cam.ac.uk/data_request/cif.

Supplementary Information accompanies this paper at http://www.nature.com/ naturecommunications

Competing financial interests: The authors declare no competing financial interests.

Reprints and permission information is available online at http://npg.nature.com/ reprintsandpermissions/

How to cite this article: He, H. et al. Polarized three-photon-pumped laser in a single MOF microcrystal. Nat. Commun. 7:11087 doi: 10.1038/ncomms11087 (2016)

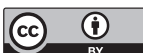

This work is licensed under a Creative Commons Attribution 4.0 International License. The images or other third party material in this article are included in the article's Creative Commons license, unless indicated otherwise in the credit line; if the material is not included under the Creative Commons license, users will need to obtain permission from the license holder to reproduce the material. To view a copy of this license, visit http://creativecommons.org/licenses/by/4.0/ 